\title{
$\mathrm{H} \alpha$ line profiles for a sample of supergiant $\mathrm{HII}$ regions
}

\section{Model line profiles}

\author{
M. Rozas, M. G. Richer, W. Steffen, G. García-Segura, and J. A. López
}

\begin{abstract}
Instituto de Astronomía, Universidad Nacional Autónoma de México, Ensenada, CP 22800, Baja California, Mexico e-mail: [maite;richer;wsteffen;ggs; jal]@astrosen.unam.mx
\end{abstract}

Received 23 March 2006 / Accepted 15 June 2006

\section{ABSTRACT}

\begin{abstract}
We carried out a series of 1D hydrodynamical computations with the ZEUS fluid solver in order to reproduce the salient features in our high-resolution $\mathrm{H} \alpha$ emission line profiles of a sample of HII regions (see Rozas et al. 2006a,b). Four models were computed. In the first and second cases, an ionization-bounded HII region was modeled with and without a shell produced by stellar winds. In the third and fourth cases, a density-bounded HII region was considered, both with and without a shell driven by stellar winds. The resulting line profiles have been modeled using the SHAPE rendering program. We find that our observed high-quality profiles are well-reproduced in the density-bounded model that includes an expanding shell formed as a result of the stellar winds from the ionizing stars. Finally, we consider the effects that the finite slit widths used for the observations have on the physical conditions deduced from the line profile of the ionized bubble.
\end{abstract}

Key words. ISM: HII regions - ISM: kinematics and dynamics - stars: winds, outflows

\section{Introduction}

The study of the kinematics and dynamics of the ionized gas in giant HII regions is of fundamental importance for understanding the mechanism of star formation better in these complexes, as well as the processes related to the interaction between massive star clusters and the surrounding gas. Giant HII regions are characterized by their high $\mathrm{H} \alpha$ luminosity $\left(10^{39}-10^{41} \mathrm{erg} \mathrm{s}^{-1}\right)$ and large dimensions (up to $1 \mathrm{kpc}$ ). In addition, they present characteristic kinematic features, such as supersonic widths in their emission line profiles, which differentiate them from HII regions known as "normal".

These supersonic movements in giant HII regions were first noted by Smith \& Weedman (1970). Later studies found correlations between different physical properties of the regions, such as luminosity and size, with their velocity dispersions (Terlevich \& Melnick 1981; Hippelein 1986; Roy et al. 1986; Melnick 1977). Specifically, Terlevich \& Melnick (1981) found that the supersonic widths of the lines are correlated with the luminosity of the regions by a law $L \sim \sigma^{4}$, similar to globular clusters and elliptical galaxies. This lead them to the conclusion that the systems are gravitationally bounded and that the width of their line profiles reflects their virialization. In that model, called the "gravitational model", Terlevich \& Melnick (1981) propose that a large number of ionized gas condensations are moving supersonically in the virialized system of gas + stars.

Although this model found support from various studies at that time, (Hippelein 1986; Roy et al. 1986; Melnick et al. 1987) a problem is encountered in explaining the replenishment of fragments of ionized gas, given that the champagne effects should disperse the gas condensations in a much shorter time than the median lifetime of the HII regions (Tenorio-Tagle et al. 1996). It is clear that an energy source that balances the dissipation caused by the radiative cooling behind the shock waves that result from these supersonic movements is necessary. This has lead to what are called "hydrodynamic models" in which it is proposed that it is the mechanical energy from thousands of ionizing stars in the clusters, transmitted by winds or supernova explosions, that induces these turbulent movements (Dyson 1979; Rosa \& Solf 1984; Hippelein \& Fried 1984; Hunter 1994; Chu \& Kennicutt 1994). The principal drawback of these models is having to calculate the number of stars necessary to explain the supersonic widths given the low conversion efficiency of the involved energies.

In recent times, a growing number of giant extragalactic HII regions have been found whose line profiles include highvelocity, low-intensity components situated symmetrically on both sides of a more intense and narrow central component. When the signal-to-noise ratio permits, the detailed analysis of these profiles shows that these high-velocity components extend for distances of hundreds of parsecs from the ionizing cluster. Many studies appear in the literature that show the existence of these high-velocity components (Chu \& Kennicutt 1994; Izotov et al. 1996; Peimbert et al. 1991; Castañeda et al. 1990), but their clear interpretation is complicated by the combination of the high velocities, low densities, and large sizes of the emitting regions. Roy et al. $(1991,1992)$ discard the possibility that these components arise as a result of instrumental effects and put forward various possibilities: Thomson scattering by hot gas, stellar winds, remnants of supernovae, or blowout of superbubbles. However, they recognize that all interpretations have problems explaining the line widths and the injected energy required to produce them. The most fruitful attempts have linked them to manifestations of expansive gas motions produced by different mechanisms. Drissen et al. (1993) consider the possibility of the ventilation of the superbubble of gas within the galactic halo as the mechanism responsible for the components found in NGC 2363. Izotov et al. (1996) propose that the wings are produced by a large number of supernova remnants $\left(\sim 10^{4}\right)$, but the 
result is not compatible with the physics of the shock waves. More recent statistical studies considering a much larger number of HII regions have been carried out by Rozas et al. (1998), Relaño \& Beckman (2005), Rozas et al. (2006a, henceforth, Paper I), and conclude that the presence of these components, henceforth denoted as wings, are a general phenomenon in the most luminous HII regions. They focus their interpretation of these line profiles on the expansion of bubbles produced by stellar winds that originate from massive stars, analyzing whether this process is capable of generating the observed energies.

The brightest complex of HII regions in M 101, NGC 5471 (Muñoz-Tuñón 1994), contains five gas condensations surrounded by an halo of diffuse gas. Analysis of the structure of the emission lines shows that all the profiles are supersonic. There is also a "splitting" of the lines detected on the borders of the nebulae, which could correspond to the components that we have previously denominated wings. On the other hand, kinematic studies of NGC 604 (Sabalisck et al. 1995; Yang et al. 1996) show that there are zones in which high velocity dispersions are associated with low "surface brightness", although gravity could be responsible for the width of the lines in the greater part of the region. These are related to a series of shells and filaments produced by the action of the stellar winds from massive stars in the region. In NGC 2363, Roy et al. (1991) find a splitting of the [OIII] line and attribute this to the presence of a bubble with a diameter of $200 \mathrm{pc}$ and an expansion velocity of $45 \mathrm{~km} \mathrm{~s}^{-1}$. Muñoz-Tuñón et al. (1996) analyze the kinematics of NGC 604 and NGC 588 and propose that the relation between velocity dispersion and surface brightness, $\sigma-I$, is an excellent diagnostic to separate the different broadening mechanisms affecting the profiles, motions produced by shells and filaments generated by massive stars, on the one hand, and gravitational broadening, on the other. They discuss different evolutionary stages for the HII regions as an explanation of the different types of broadening.

Capriotti \& Kozminski (2001) analyze the effects of the stellar winds from the ionizing stars on the structure and dynamics of HII regions. They conclude that photoionization dominates, as winds with the strengths expected for zero-age O-type stars have little influence on the ultimate overall structure and dynamics of radiation-bounded H II regions, except for the case of wind models featuring nebulae of very high density. According to these authors, even though heating by photoionization seems to dominate the evolution of $\mathrm{HI}$ regions and the formation of $\mathrm{H}$ II regions surrounding O-type stars, dense molecular clouds may be more strongly influenced by winds. Likewise, they argue that winds can also be more influential in the overall structure and dynamics of gas-bounded $\mathrm{H}$ II regions of moderate density if the system is sufficiently optically thin to ionizing radiation.

The first attempt to relate the width of the lines with the density-bounded regime of the HII regions is the statistical study carried out by Rozas et al. (1998) on the HII regions of the galaxy M 100, in which the relationship between the velocity dispersion and luminosity of the HII regions was analyzed. They conclude that the upper envelope occupied by HII regions in the luminosity-velocity dispersion plane, L- $\sigma$, are virialized and density-bounded. This result is consistent with two subsequent, similar studies carried out by Relaño et al. (2005) and in Paper I.

In Paper I, we reported the observations of a homogeneous set of high-quality echelle spectra, obtained at a resolution of $\sim 11 \mathrm{~km} \mathrm{~s}^{-1}$, for a sample of giant HII regions. The line profiles in the data show the systematic presence of extended components that our spectra are able to resolve. Rozas et al. (2006b, henceforth, Paper II), analyzed the wings found in the line

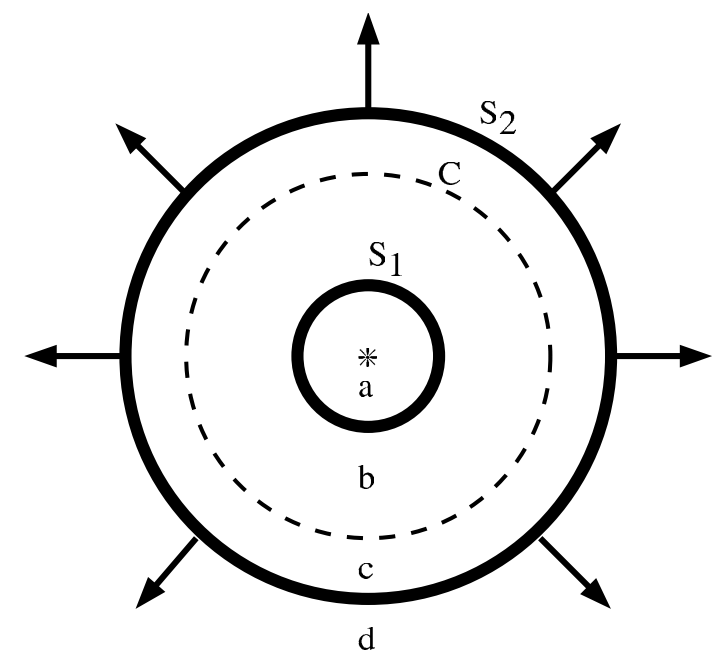

Fig. 1. Scheme of the interaction between the stellar winds emitting by ionizing stars and the interstellar medium of the HII region. See description of each zone in the text.

profiles of this sample of giant HII regions and calculated the energies involved, assuming that the wings are the kinematic signature of an expanding shell whose formation is the result of stellar winds from the ionizing stars. They concluded, based upon the premise of density-bounded HII regions, that the mechanical energy of the winds is enough to produce the kinetic energy of the observed shells. Here, we reproduce the observed profiles using models that span the luminosity- and density-bounded regimes and including shells produced by winds. We analyze the effects of inhomogeneities in the hydrogen distribution within the HII region and those stemming from the variation in size of a simulated slit used for making the observations.

\section{Feasible driving mechanisms}

The massive clusters of stars that photoionize giant extragalactic HII regions develop strong winds that generate a large quantity of mechanical energy capable of upsetting the medium surrounding the cluster. The interaction between the winds and the ISM produces a thin shell of swept-up interstellar medium with less dense material in its interior. In the interaction, 4 zones can be identified (see Fig. 1), and its description can be found in Dyson \& Williams (1980), who assume a separation of shocked wind gas and shocked interstellar gas by a contact surface (line $\mathrm{C}$ in Fig. 1) and propose no transfer of heat between the two zones. Weaver et al. (1977) allow for conductive heat flow across the surface. In the first case radiative cooling of region (b) is ignored because the density is low and the temperature high. It is the dense region (c) that is radiatively cooled to a temperature $\sim 10^{4} \mathrm{~K}$, and this temperature is maintained by the ionizing radiation from the stars. The thin shell is driven by the pressure of hot wind gas at a higher temperature. In the second case, conductive heat flow though the contact surface (Weaver et al. 1977) cools the gas in the region (b). In either case, during the interaction of the stellar winds with the ISM, a fraction of the wind's kinetic energy is converted into kinetic energy of the ISM and is used to move the shell of swept-up material. When the wind and the ISM mix and the radiative losses are large, the energy available to move the shell is reduced, given that some fraction of the wind's energy is lost by radiation (Capriotti \& Kozminski 2001). 


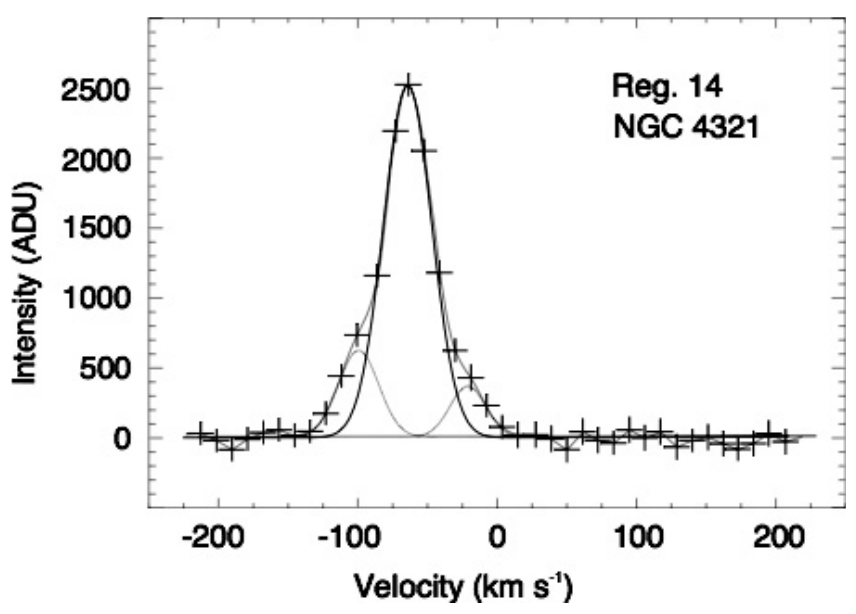

Fig. 2. $\mathrm{H} \alpha$ line profile for one of the HII regions in our sample (Paper I). We can see the principal component and the two wings: the broad, low intensity and high velocity components.

Applying the principle of the conservation of momentum and energy to the bubble in phase (3), Pikelner (1968) and Dyson \& de Vries (1972) find a solution for the radius of the shell:

$R_{\mathrm{S}}(t)=0.76\left(\frac{\dot{E}_{\mathrm{W}} t^{3}}{\rho_{0}}\right)^{1 / 5}$

where $\dot{E}_{\mathrm{W}}=\frac{1}{2} \dot{M}_{\mathrm{W}} v_{\mathrm{W}}^{2}$ and $\rho_{0}$ is the density of the interstellar gas. With this solution, the kinetic energy of the shell is:

$E_{\mathrm{k}}=0.20 \dot{E}_{\mathrm{W}} t$.

This result was used in Paper II to conclude from the observed line profiles that the energies provided by the winds are sufficient for producing the observed features in the spectral profiles.

\section{The data}

The profiles that we wish to reproduce are those analyzed in Papers I and II. In those studies, the brightest and most isolated HII regions from a series of spiral galaxies were observed with high resolution spectroscopy, and the great majority of them were found to present line profiles with components of high velocity and low intensity that were attributed to the action of the stellar winds of the ionizing stars. More details regarding the observations and the profiles may be found in Papers I and II. A characteristic profile is shown in Fig. 2.

\section{Numerical simulations}

We have computed four simple examples that can serve as a guide to understanding the kinematics of giant HII regions. These sets of $1 \mathrm{D}$ hydrodynamical computations were done with the magneto-hydrodynamic fluid solver ZEUS-3D (version 3.4; Clarke 1996). This code is the updated, 3D version of the twodimensional code ZEUS-2D (Stone \& Norman 1992), a Eulerian explicit code that integrates the equations of hydrodynamics for a magnetized ideal gas. With the proper symmetry axis, it works efficiently in one dimension.

ZEUS-3D does not include radiation transfer, but we implemented a simple approximation to derive the location of the ionization front for arbitrary density distributions (see Bodenheimer et al. 1979). This is done by assuming both that ionization equilibrium holds at all times and that the gas is fully ionized inside the HII region. The position of the ionization front is given by $\int n^{2}(r) r^{2} \mathrm{~d} r \approx F_{\star} / 4 \pi \alpha_{B}$

The models include the Raymond \& Smith (1977) cooling curve above $10^{4} \mathrm{~K}$. For temperatures below $10^{4} \mathrm{~K}$, the shocked gas region is allowed to cool down with the radiative cooling curves given by Dalgarno \& McCray (1972) and MacDonald \& Bailey (1981). Finally, the photoionized gas is always kept at $10^{4} \mathrm{~K}$, so no cooling curve is applied to the HII regions (unless, of course, there is a shock inside the photoionized region). We perform the simulations in spherical coordinates with 1000 zones in the radial direction and a physical size of $400 \mathrm{pc}$. The clusters are modeled for simplicity as the sum of $60(\mathrm{O} 3(\mathrm{~V}))$ stars, giving a total $\dot{M}=2.76 \times 10^{5} M_{\odot} \mathrm{yr}^{-1}$ and a total number of ionizing photons $F_{\star}=4.38 \times 10^{51} \mathrm{~s}^{-1}$. The stellar winds from the clusters is set as usual: several computational zones at the origin of the grid are set with the wind conditions, $\rho=\dot{M} / 4 \pi r^{2} v_{\infty}$, and $v_{\infty}=3000 \mathrm{~km} \mathrm{~s}^{-1}$. Also, $\dot{M}$ and $v_{\infty}$ are related to the mechanical luminosity $L_{\mathrm{w}}=1 / 2 \dot{M} v_{\infty}^{2}$.

In our study, we considered a homogeneous ISM as the initial condition, which supplies the external pressure. The diffuse ISM is thought to consist of four major components: the cold neutral medium (CNM), the warm neutral medium (WNM), the warm ionized medium (WIM), and the hot ionized medium (HIM). The WNM, is, by far, the ISM component that occupies much of the space (Kulkarni \& Heiles 1988) in an "intercloud medium" and surrounds the cold clouds. However, the WNM is the least understood component of the ISM, since the information comes mainly from $21 \mathrm{~cm}$ line emission data and direct measurements of temperatures (estimated to be in the range $5000 \mathrm{~K}$ to $7000 \mathrm{~K}$ ) or densities (between 0.1 and $1 \mathrm{~cm}^{-3}$ ) are difficult to obtain. Thus, in order to have thermal pressures of $P / K=1 \times 10^{4} \mathrm{~cm}^{-3} \mathrm{~K}$, we adopted $n \sim 1.67 \mathrm{~cm}^{-3}$ for the ISM density and $6000 \mathrm{~K}$ for the temperature. This gives us a sound speed of $c_{\text {slisot }} \sim 7 \mathrm{~km} \mathrm{~s}^{-1}$.

We computed four models (Fig. 3). The first two are examples of ionization-bounded regions, while the second pair are density-bounded, for which a density jump of three orders of magnitude was included at $100 \mathrm{pc}$ from the cluster. This jump simulates the end of the HI cloud.

Figure 3 shows the four models at 1 Myr of their evolution. The first model (S1) is an HII region without stellar winds. The ionizing front is located at $370 \mathrm{pc}$. The second model (S2) is a HII region with stellar winds in which a superbubble has reached a radius of $55 \mathrm{pc}$. The third and fourth models (M1 and M2) are equivalent to the first and second, respectively, but including a density jump at $100 \mathrm{pc}$ in the ISM, which produces a "champagne" flow of low density and high velocity (Tenorio-Tagle et al. 1979).

\section{Model line profiles}

Spatially-resolved model line profiles from the spherically symmetric hydrodynamic simulations were produced using the SHAPE code (for a detailed description see Steffen \& López 2006). SHAPE produces position-velocity diagrams and $1 D$ line profiles from a distribution of moving particles. It is used to model the 3D-structure and kinematics of complex gaseous nebulae. Here we have used the $1 \mathrm{D}$ results from the hydrodynamic simulation as an input to SHAPE. We modeled a sphere with a constant number of particles for each of the 1000 grid-points of the simulation in SHAPE. The particles move radially with the velocity given by the simulation. The brightness of the particles is given by the emission measure (EM) of the simulation. Since a constant number of particles was used for all radii $r$, the emission 


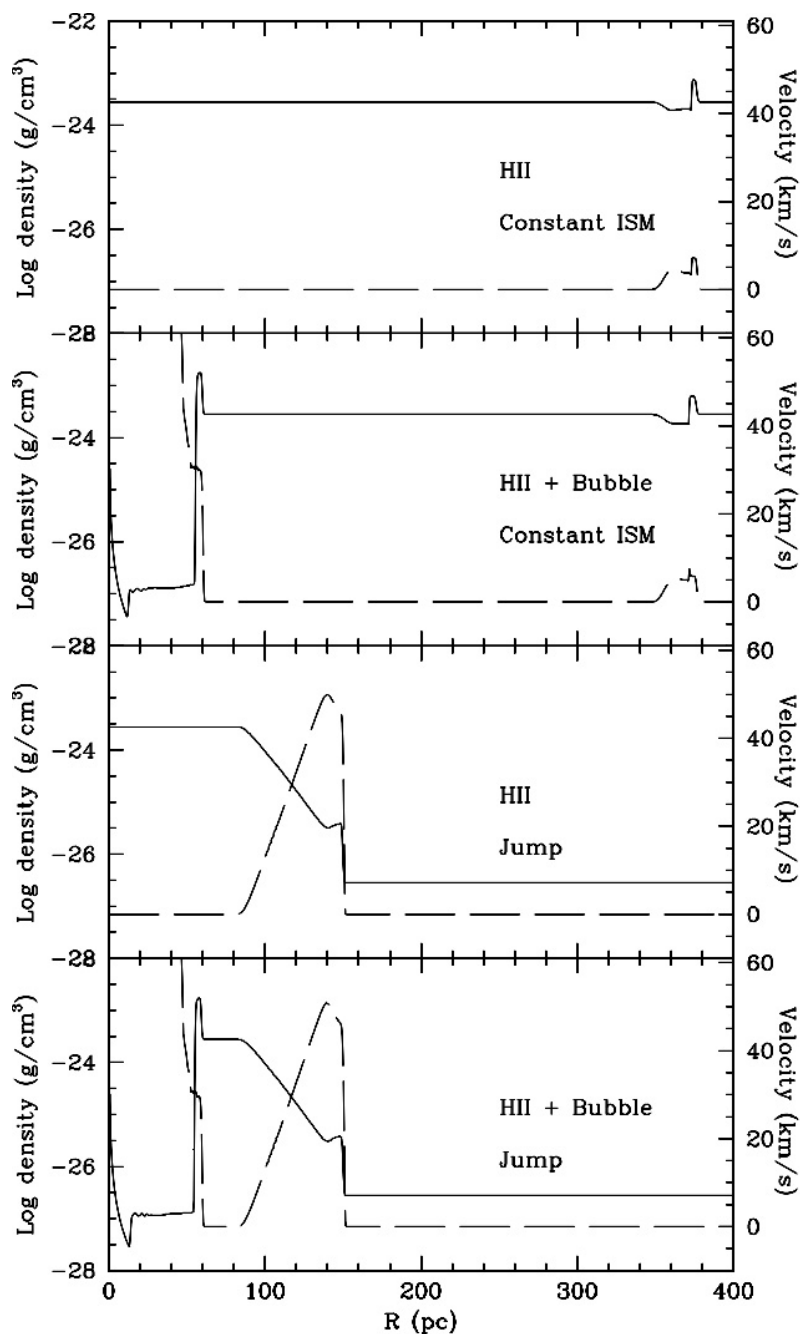

Fig. 3. Snapshots at $1 \mathrm{Myr}$ of the evolutions. The gas densities are plotted with solid lines, while the radial velocities are plotted with long dashed lines. The first model (top) is a HII region without stellar winds. The second model (middle-top) is a HII region with stellar winds. Both models are ionization-bounded. The third model (middle-bottom) is an HII region with a density jump in the ISM. The fourth model (bottom) is an HII region with stellar winds and the same density jump. The jump was originally located at $100 \mathrm{pc}$. The last two models are density-bounded.

from each particle was scaled with the surface area $4 \pi r^{2}$. Three hundred particles where used at random positions with a different distribution for each sphere. The total number of particles for the full simulation is then $3 \times 10^{5}$, which ensures an adequate sampling to produce symmetric line profiles to within a few percent, as expected from a spherically symmetric simulation.

In the modeling of these line profiles we assumed that the slit width covers the complete simulation, which is equivalent to a slitless spectrum. A velocity resolution of $20 \mathrm{~km} \mathrm{~s}^{-1}$ was assumed, i.e. the raw-spectra were convolved with a gaussian with a $F W H M$ of $20 \mathrm{~km} \mathrm{~s}^{-1}$. Models M1 and S1 do not show any high-velocity wings at levels greater than 0.1 percent of the line peak, and they are completely dominated by the stationary HII-region (Fig. 4). The opposite case is model S2, where almost no stationary material is present, and the emission is dominated by the expanding superbubble. Hence a plateau-like line profile characteristic of a thin expanding shell is found with only a small central peak (Fig. 4).

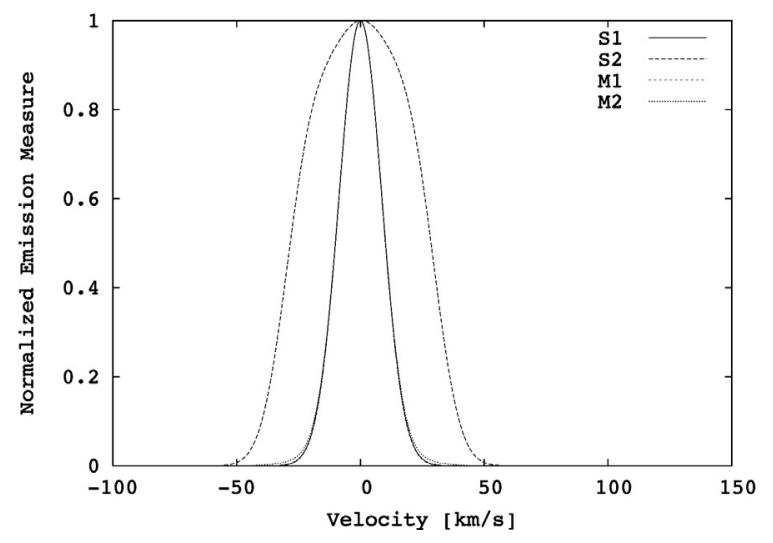

Fig. 4. The variation in line profiles for the different hydrodynamical models.

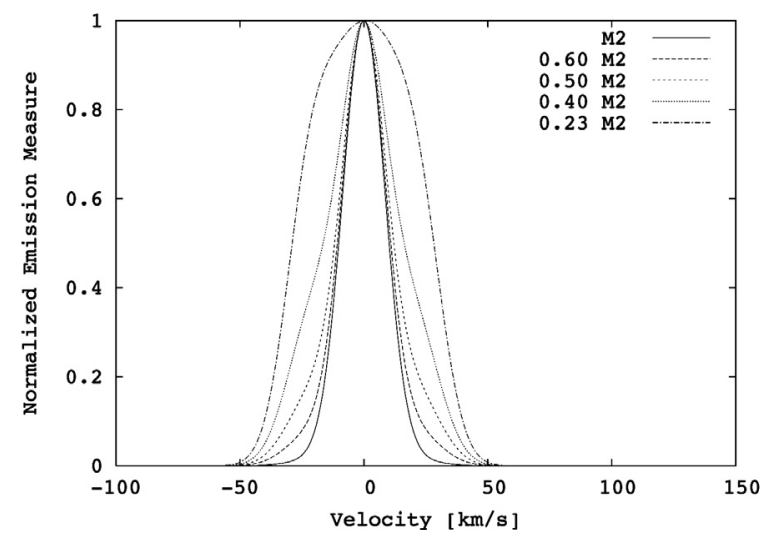

Fig. 5. The variation in line profiles with size ratio between HII-region and superbubble for model M2: a density bounded HII region with a expansive shell produced by the stellar winds.

Only model M2 can produce line profiles similar to those observed. In Fig. 5, we show the profiles obtained using the hydrodynamical model M2, varying the ratio between the sizes of the HII region and the shell. This agrees with the expectation that the ratio of the emission from the high-velocity wings and from the stationary HII-region is proportional to the ratios of their densities and their volumes. The emission line profile deduced from the full volume of simulation M2 shows very weak high velocity wings compared to the observed ones. However, assuming that the HII-region is density-bounded at a distance smaller than the $400 \mathrm{pc}$ assumed in the simulation, the peak of the stationary volume is considerably reduced (Fig. 5). For the simulated superbubble to produce the observed peak to wing ratio, the radius of the quiet HII-region should be approximately 3 to 4 times that of the superbubbles.

Since we are using only emission measure values and not the true line emission, this value can be expected to vary. This value may also vary because the hydrodynamic simulation has a limited gas compression in the shell of the superbubble. If the true compression is higher, then the ratio of radii between the superbubble and HII-region is higher in the same proportion; since the emission coming from the superbubble is proportional to the square of the density and for the HII-region, it is proportional to the square of its radius. 


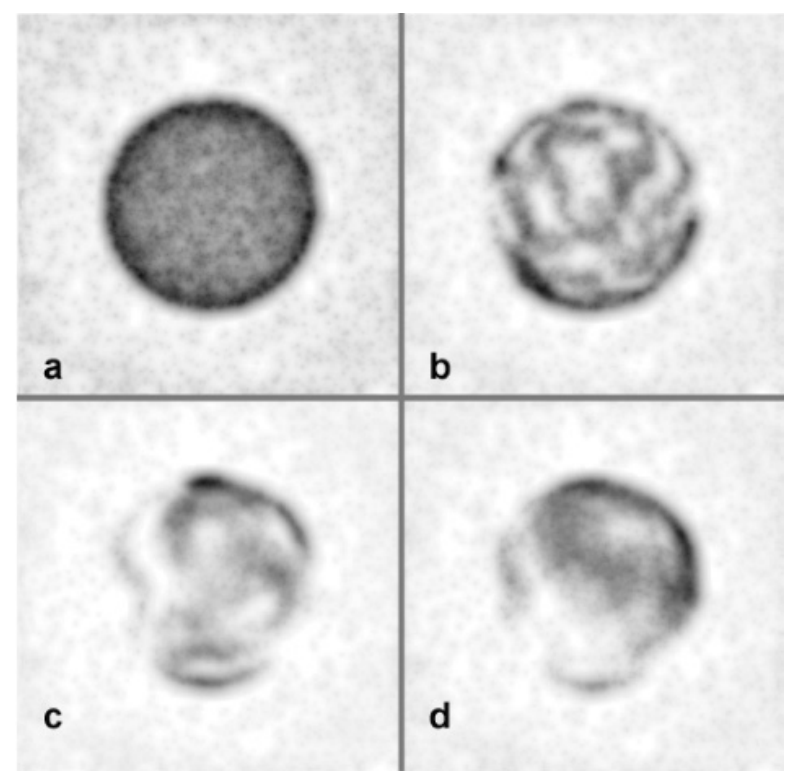

Fig. 6. The variation in the images for different structural noise size of the superbubble are shown. In images a to d, the noise size has values of $0.0,0.6,1.4$, and 2.2 times the radius of the bubble. The brightness is normalized to the peak brightness and displayed such that the low brightness features are visible. The graininess of the images is due to the resolved random positions of the individual particles. Only the neighborhood of the bubble shell is shown.

\subsection{The structure of the superbuble}

Using SHAPE to explore the origin of the asymmetries of the wings of the observed line profiles, we constructed a series of morphological models of a superbubble with varying filamentary surface structures expanding in an inert ambient medium.

First we introduced a noise pattern with different sizes that produces the filamentary structure of the superbubble as shown in Fig. 6. The typical size of the filaments (noise size) is similar to the radius of the bubble. In images 6 a to $6 \mathrm{~d}$ the noise size has values of $0.0,0.6,1.4$, and 2.2 times the radius of the bubble, and the brightness is normalized to the peak brightness. In Fig. 7 we compare the corresponding line profiles. We chose a brightness ratio between the stationary HII-region and the shell similar to the intensity ratio between the main components and the fast and wide ones observed in the lines profiles. The expansion speed of the bubble is $50 \mathrm{~km} \mathrm{~s}^{-1}$ with a random variation of $10 \mathrm{~km} \mathrm{~s}^{-1}$ to simulate turbulence. The ambient medium is stationary on average but has a turbulent variation of $20 \mathrm{~km} \mathrm{~s}^{-1}$. The line profiles are rendered with a velocity resolution of $20 \mathrm{~km} \mathrm{~s}^{-1}$. The number of particles used for the superbubble is 1500 , and there are 8500 for the ambient medium. The size ratio between the ambient medium and the bubble is 2.5 and the region inside the bubble is empty. The ratio of total emission measure between the bubble and the ambient medium is 1.75 .

As this simulation shows, the observed asymmetries can be explained if significant variation in surface brightness is present in the superbubble. The orientation of these filamentary structures may have a profound effect on the line shape. In Fig. 8 we show the change in line shape for one of the filamentary superbubbles only due to different orientations in space.

We find that for the same bubble structure some line profiles are almost symmetric whereas others are highly asymmetric. This shows that a detailed interpretation of the one-dimensional line profiles gives little information about the structure of the superbubble.

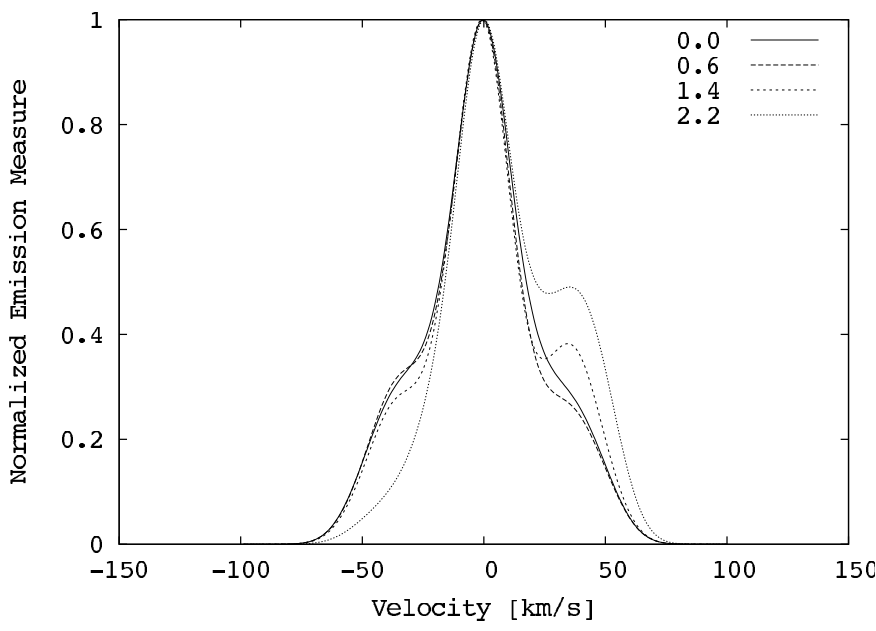

Fig. 7. The variation in the line profiles for different structural noise sizes of the superbubble is shown, which corresponds to the images in Fig. 6.

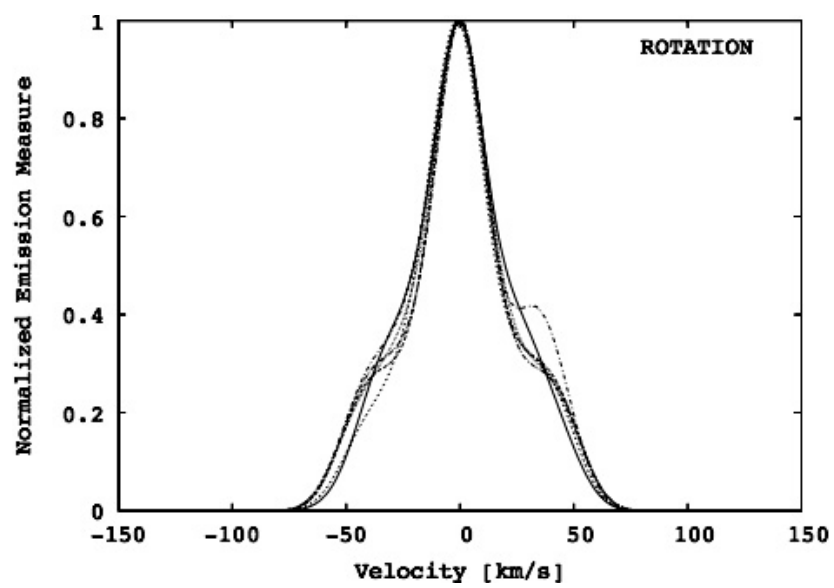

Fig. 8. The variation in line profiles for various orientations of the superbubble is compared. We show the profiles obtained for 6 different orientations of the filamentary shell with a noise size of 0.6. Some profiles are nearly symmetric, while others are strongly asymmetric even for the same bubble structure.

\section{The problem of resolution}

In this section we analyze the influence of the size of the slit on the object and its effects on the form of the observed profiles. These effects are illustrated in Fig. 9, which shows the profile calculated for an artificial region, constructed with the hydrodynamic model M2, and observed with slits of different widths. The profiles were constructed using an HII region with a diameter of 900 units and shell diameter of 300 units. The slits, in the same units, range from a width of 1000 , which covers the entire region, to a central slit with a width of 30 units, which resolves the shell perfectly, passing through various intermediate values.

In addition, the expected profiles from the observation of an expanding shell have been calculated without any contribution from the HII region, with the slits previously mentioned. These profiles appear in Fig. 10. In each case, the velocity of the shell is $50 \pm 2 \mathrm{~km} \mathrm{~s}^{-1}$ with a resolution in velocity of $20 \mathrm{~km} \mathrm{~s}^{-1}$. For a determined velocity resolution $\left(20 \mathrm{~km} \mathrm{~s}^{-1}\right.$ in our case), we can establish a "maximum" profile (case (a) of Fig. 10), which is constructed using the widest slit; but this does not resolve the shell and instead gives us the contribution of the shell to the principal component of the total profile of the HII region and 


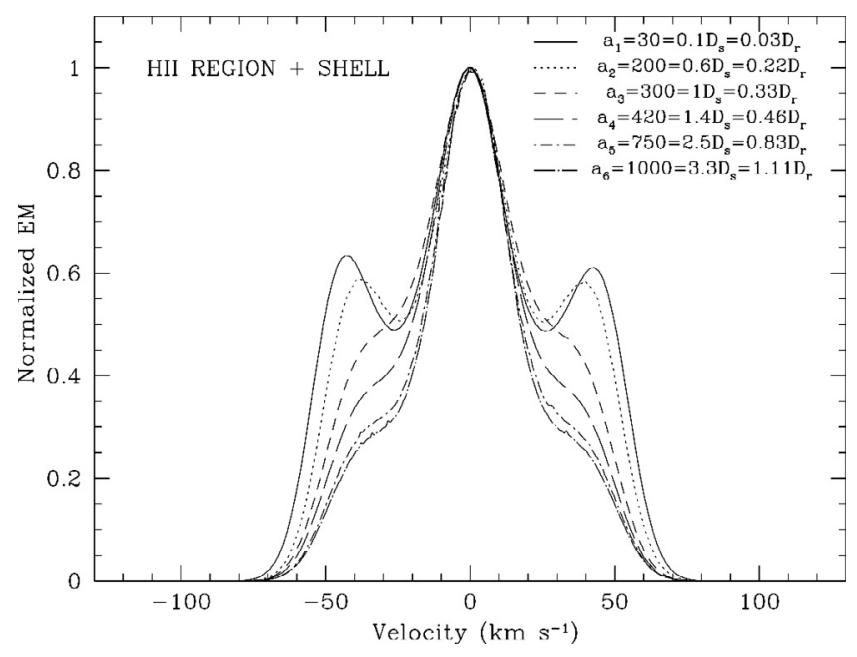

Fig. 9. Line profiles obtained using the hydrodynamic model M2 supposing that the observations are made with slits of differing widths. As indicated in the upper right of the panel, different line styles denote the slit widths (apertures) of different sizes in instrumental units (from 30 to 1000 units). The legend also gives the ratio of these slit widths to the sizes of the shell $\left(D_{\mathrm{s}}\right)$ and the HII region $\left(D_{\mathrm{r}}\right)$. The different profiles obtained with the slits of smallest to largest size have been fit with three Gaussians whose parameters correspond to the fits in Table 1.

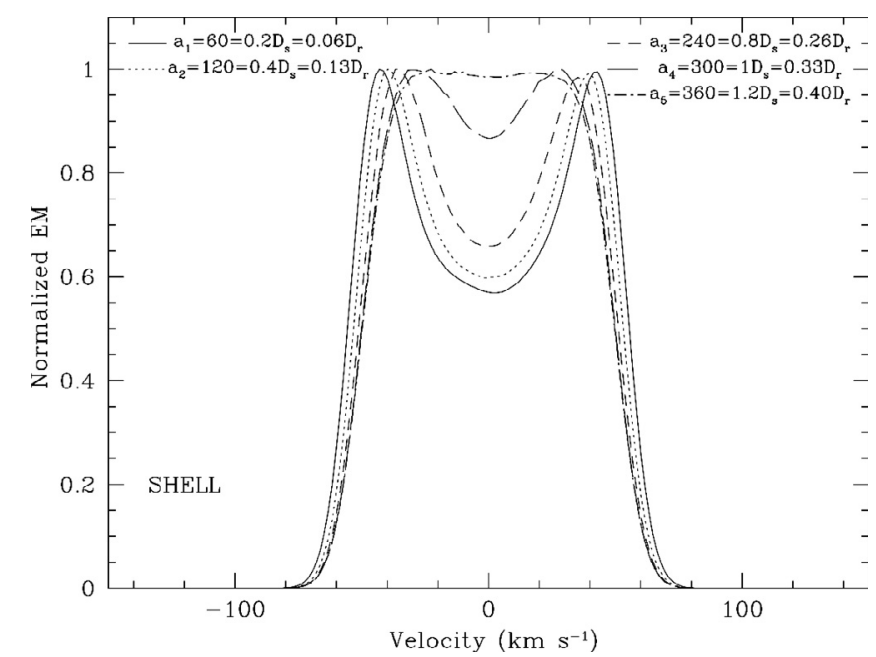

Fig. 10. Line profiles of a shell in expansion, without consideration of the contribution of the HII region, supposing that the observations are made with slits of differing widths. As in the previous figure, we note the size of the slits with respect to the size of the shell $\left(D_{\mathrm{s}}\right)$ and to the size of the HII region $\left(D_{\mathrm{r}}\right)$. The different profiles obtained with the slits of smallest to largest sizes correspond to the fits in Table 2.

a "mimimum" profile (case (d) in Fig. 10), which corresponds to the slit that resolves the shell and that shows the "dip" in the flux in the center of the profile. In the intermediate cases, the "dip" in flux diminishes with the increase in width of the slit.

For comparison's sake, we show the artificial profiles in Fig. 11 obtained in the theoretical case of a "infinite" resolution in velocity. In this figure we represent the profiles of the HII region with an expanding shell, with the same sizes and velocities as those used to reproduce the profiles in Fig. 10, observed with a slit that includes the entire object and with a very narrow slit that resolves the shell perfectly, both cases with a very high resolution in velocity. Similarly we represent the theoretical profiles of the isolated shell in both situations. As expected, a flat profile is obtained in the case of a wide slit that covers the entire shell and

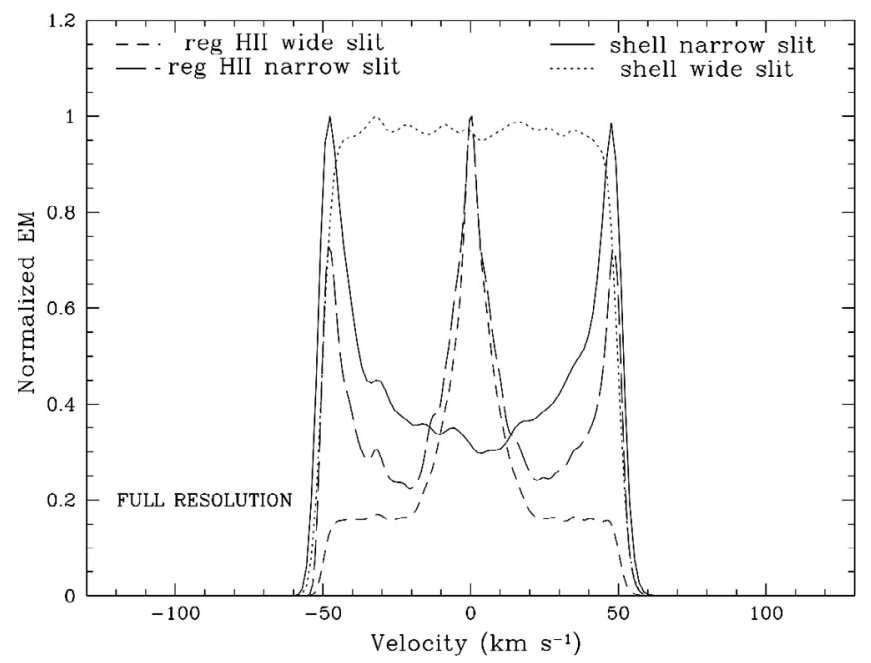

Fig. 11. Artificial profiles obtained in the theoretical case of an infinite resolution in velocity. The different profiles are discussed in the text.

a "U"-shaped profile when using the narrow slit. In these cases the deviations of the flat profile and the small widening that is observed in the peaks of the profile with the form "U" are due to the variation in velocity of $2 \mathrm{~km} \mathrm{~s}^{-1}$ and to the result of averaging every 3 pixels in velocity in order to diminish the numerical noise introduced by the random distribution of a limited number of particles.

In Tables 1 and 2 we present the results of fitting the profiles with multiple Gaussian components. The models $r_{1}, r_{2}, \ldots$ $r_{6}$ in Table 1 are the fits corresponding to Fig. 9, in other words, to the case of the HII region + shell for the different slit widths previously mentioned, from smallest to largest. The models denominated $c_{1}, c_{2}, \ldots c_{5}$ of Table 2 are the corresponding fits to Fig. 10, the model of the isolated shell, without the contribution of the HII region for the different widths slits indicated in the figure, also from smallest to largest. For each fit we show the central values in velocity of each component (Col. 2), the fraction of the total flux (in \%) (Col. 3) and the FWHM of the component (Col. 4). For each fit, the first two components have the highest velocities, which corresponds in the real cases to the high velocity components that we denominate wings. In the case of profiles for an HII region with an expanding shell, we fit three Gaussians, one for the HII region itself and two for the wings, as was done for the observations in Papers I and II. In the cases of the profiles of a shell without a surrounding HII region, the profiles were fit with a number of Gaussians that reproduce them best and we show only the values in Table 2 corresponding to the two Gaussians of the highest velocity, i.e., the two peaks of each profile.

As observed in Figs. 9 and 10, there are various important effects to be considered. Consider first the case of an expanding shell without a surrounding HII region. Adopting $50 \mathrm{~km} \mathrm{~s}^{-1}$ as the true expansion velocity (the velocity of the shell with which the artificial profiles were obtained), the position of the peaks in the wings generally underestimates this velocity, because the separation between them is only a good measure of the expansion velocity if the shell is completely resolved. The deduced expansion velocity diminishes as the fraction of the shell in the slit increases, and, once the slit includes the entire shell, the deduced expansion velocity does not change. In fact, the half width at half maximum (HWHM) of the profiles for the shell are a better estimation of the expansion velocity if the shell is not resolved or is only partially resolved, as observed in Col. 5, Table 1, where 
Table 1. Parameters of the fits of the emission profiles corresponding to Fig. 9, calculated with an artificial region, constructed from model M2, and observed with slits of different widths.

\begin{tabular}{ccccc}
\hline \hline Model & Velocity $\left(\mathrm{km} \mathrm{s}^{-1}\right)$ & $E M(\%)$ & $F W H M$ & $E M(\text { wings })_{50}$ \\
\hline \multirow{2}{*}{$\mathrm{r}_{1}$} & -42.8 & 17.4 & 26.4 & \\
& +42.5 & 17.2 & 27.1 & 0.84 \\
& -0.4 & 39.6 & 37.9 & \\
$\mathrm{r}_{2}$ & -38.9 & 16.3 & 27.0 & \\
& +39.8 & 16.3 & 27.1 & 0.61 \\
& +0.6 & 36.5 & 35.0 & \\
\hline \multirow{2}{*}{$\mathrm{r}_{3}$} & -35.2 & 14.7 & 31.3 & \\
& +35.0 & 14.7 & 31.4 & 0.54 \\
& -0.03 & 33.9 & 32.6 & \\
$\mathrm{r}_{4}$ & -35.1 & 11.7 & 31.0 & \\
& +35.2 & 11.4 & 31.0 & 0.53 \\
& -0.0 & 32.8 & 31.4 & \\
$\mathrm{r}_{5}$ & -34.9 & 9.3 & 30.2 & \\
& +35.9 & 9.1 & 30.3 & 0.52 \\
& +0.6 & 31.9 & 30.3 & \\
$\mathrm{r}_{6}$ & -34.9 & 8.5 & 29.8 & \\
& +35.9 & 8.4 & 30.2 & 0.50 \\
\hline
\end{tabular}

we show the relative intensity of the wings corresponding to an expansion velocity of $50 \mathrm{~km} \mathrm{~s}^{-1}$ (cf. Gesicki \& Zijlstra 2000, for similar results). As Table 2 illustrates, the expansion velocity deduced from the positions of the profile peaks can underestimate the true expansion velocity by up to $30 \%$.

It is notable that, for a given slit width, the properties of the shell do not depend upon whether the HII region is present. In other words, the HII region does not confuse the measurement of the shell properties. In the four cases of the Fig. 9 when the slit covers the entire shell (slits $\mathrm{a}_{3}, \mathrm{a}_{4}, \mathrm{a}_{5}$ and $\mathrm{a}_{6}$ ), the expansion velocities measured for the shell are the same despite their variable contribution to the total profile.

Another important effect concerns the fluxes attributed to the shell and the HII region. Unless the slit completely resolves the shell, significant emission from the shell (wings) overlaps the velocity range occupied by the HII region (central peak); i.e. in order to determine the total emission from the expanding shell, it is insufficient to fit two Gaussian high-velocity wings. Instead, the flux in the high-velocity wings represents only slightly more than half of the total flux from the shell. Considering the models of the shell alone, the Gaussian components corresponding to the highest velocities (positive and negative) contain an almost constant fraction of the total flux, varying from $54.4 \%$ to $58.5 \%$.

The last notable result related to the slit width is that the presence of the shell affects the properties deduced for the HII region. The input HII region profile width was $28.28 \mathrm{~km} \mathrm{~s}^{-1}$, but a higher value is always measured. This increase is due to the emission from the shell with velocities that overlap those of the HII region. The most reliable measurement of the width of the profile from the HII region is obtained using a slit that covers the entire object. When narrow slits are used, the width of the HII region profile may be overestimated by some $30 \%$. Consequently, large spectroscopic apertures are favored for observations of the HII region's kinematics, e.g., with Fabry-Perot instruments, whereas narrow apertures are favored for the most reliable measurements of the shell's properties.

The foregoing implies two important changes in the interpretation of the line profiles. First, computing the fluxes in the shell from those of the wings alone, and the consequent energies required to form these shells, are lower limits to the true values,
Table 2. Parameters of the fits of the emission profiles corresponding to Fig. 10, calculated for an artificial shell, without consideration of the HII region and observed with slits of differing widths.

\begin{tabular}{cccc}
\hline \hline Model & Velocity $\left(\mathrm{km} \mathrm{s}^{-1}\right)$ & $E M(\%)$ & $F W H M$ \\
\hline & -44.1 & 24.1 & 23.8 \\
$\mathrm{c}_{1}$ & +44.2 & 22.8 & 24.2 \\
\hline & -41.3 & 25.0 & 25.0 \\
$\mathrm{c}_{2}$ & +41.2 & 24.8 & 25.1 \\
\hline & -38.4 & 26.3 & 27.3 \\
$\mathrm{c}_{3}$ & +38.4 & 26.2 & 27.3 \\
\hline & -34.6 & 30.8 & 33.2 \\
$\mathrm{c}_{4}$ & +34.2 & 31.4 & 33.6 \\
\hline & -35.3 & 28.0 & 32.5 \\
$\mathrm{c}_{5}$ & +35.2 & 28.0 & 32.6 \\
\hline
\end{tabular}

which are very likely almost a factor of two larger. Second, the line profile from the shell significantly perturbs the properties measured for the surrounding HII region. In particular, its velocity width is systematically overestimated. Consequently, the energies required to maintain the observed supersonic line widths are actually substantially smaller than have been supposed.

\section{Conclusions}

- We carried out a series of hydrodynamic 1D computations with the magneto-hydrodynamic fluid solver ZEUS-3D with a view to reproduce and better understand the form of the $\mathrm{H} \alpha$ emission line profiles in a sample of giant extragalactic HII regions. In these line profiles, we observe wide components, that we denominate wings, situated on both sides of a central, more intense and narrow component. We computed four models, two of which are ionization-bounded regions, and the other two density-bounded. The first model is a HII region, without considering the stellar winds produced by the ionizing stars, the second model considers these winds that trigger the formation of a shell at a certain distance from the center of the region. The third and fourth models are similar to the previous two, but with the HII regions being densitybounded. Line profiles were produced from hydrodynamical simulations using the SHAPE code. We find that the observed emission line profiles are adequately reproduced only by the model of a density-bounded HII region with an expanding shell (model M2).

- With a view to exploring the origin of the asymmetry of the wings found for some of the profiles, we constructed a series of morphological models of the shell changing its surface filamentary structure. These asymmetries can be explained if a significant variation in the surface brightness is present in the shell. The orientation of these filamentary structures modifies the shapes of the profile obtained: the same shell structure can produce profiles with different forms, symmetrical, or highly asymmetrical, depending upon its orientation. The interpretation of the one-dimensional profiles therefore provides little information regarding the structure of the shell.

- We have analyzed the influence of the spatial resolution, or the size of the slit with respect to the object, on the shape of the observed profiles. We constructed line profiles of an artificial HII region based upon model M2 and observed it with slits of differing widths. We also constructed the profiles expected from an expanding shell alone, again varying the width of the simulated slits with which the observations are made. From this analysis we conclude the following. 
- If the expansion velocity of the shell is deduced from the position of the peaks of the wings, it usually underestimates the true expansion velocity, since the separation between the peaks is a good measure of this velocity only if the shell is fully resolved spatially, in agreement with previous work. A better estimate of the true expansion velocity is obtained from the position at which the intensity of the wings is half their peak intensity when the shell is not resolved or partially resolved.

- For a given width of the slit, the properties of the shell do not vary, independent of whether an HII region is present. In other words, the HII region does not affect the measurement of the shell properties.

- A significant fraction of the flux from the shell is not considered when, in the observed profiles, we neglect the emission from the shell that overlaps the velocities occupied by the HII region. To account for this effect, the flux measured for the wings should be corrected upwards by a factor close to two, which varies slightly depending on the width of the slit.

- The presence of the emission of the shell at velocities close to zero, overlapping the velocity range of the HII region, significantly affects the velocity width measured for the HII region. The velocity width of the component corresponding to the HII region is always overestimated, with the error increasing when the shell is less resolved.

Acknowledgements. M.G.R. and J.A.L. acknowledge financial support from CONACyT grant 43121-E. M.G.R., J.A.L., and W.S. acknowledge financial support from DGAPA grants IN112103, IN108406-2, and IN108506-2. We acknowledge the work of G. Melgoza and S. Monrroy as telescope operators during the acquisition of these data.

\section{References}

Bodenheimer, P., Tenorio-Tagle, G., \& Yorke, H. W. 1979, ApJ, 233, 85 Capriotti, E. R., \& Kozminski, J. F. 2001, PASP, 113, 677 Castañeda, H. O., Vilchez, J. M., \& Copetti, M. 1990, ApJ, 365, 164
Chu, Y.-H., \& Kennicutt, R. C. 1994, ApJ, 425, 720

Clarke, D. 1996, ApJ, 457, 291

Dalgarno, A., \& McCray, R. A. 1972, ARA\&A, 10, 375

Drissen, L., Roy, J.-R., \& Moffat, A. 1993, AJ, 106, 1460

Dyson, J. E., \& de Vries, J. 1972, A\&A, 20, 223

Dyson, J. E. 1979, A\&A, 73, 132

Dyson, J. E., \& Williams, D. A. 1980, Physics of the interstellar medium (New York: Halsted Press)

Gesicki, K., \& Zijlstra, A. A. 2000, A\&A, 358, 1058

Hippelein, H. H. 1986, A\&A, 160, 374

Hippelein, H., \& Fried, J. W. 1984, A\&A, 141, 49

Hunter, D. A. 1994, AJ, 108, 1658

Izotov, Y. I., Dyak, A., Chaffee, F., et al. 1996, ApJ, 458, 524

Kulkarni, S. R., \& Heiles, C. 1988, in Galactic and Extragalactic Radio Astronomy, ed. K. Kellermann, \& G. L. Verschuur, 95

MacDonald, J., \& Bailey, M. E. 1981, MNRAS, 197, 995

Melnick, J. 1977, AJ, 213, 15

Melnick, J., Moles, M., Terlevich, R., \& García-Pelayo, J. M. 1987, MNRAS, 226, 849

Muñoz-Tuñón, C., Tenorio-Tagle, G., Castaneda, H. O., \& Terlevich, R. 1996, AJ, 112,1636

Peimbert, M., Sarmiento, A., \& Fierro, J. 1991, PASP, 103, 815

Pikelner, S. B. 1968, Astrophys. Lett., 2, 97

Raymond, J. C., \& Smith, B. W. 1977, ApJS, 35, 419

RelaÜño, M., Beckman, J. E., Zurita, A., Rozas, M., \& Giammanco, C. 2005, A\&A, 431, 235

Relaño, M., \& Beckman, J. E. 2005, A\&A, 430, 911

Roy, J.-R., Aube, M., McCall, M. L., \& Dufour, R. J. 1992, ApJ, 386, 498

Roy, J.-R., Arsenault, R., \& Joncas, G. 1986, ApJ, 300, 624

Roy, J.-R., Boulesteix, J., Joncas, G., \& Grundseth, B. 1991, ApJ, 367, 141

Rosa, M., \& Solf, J. 1984, A\&A, 130, 29

Rozas, M., Richer, M. G., \& López, J. A. 2006a, A\&A, 455, 539

Rozas, M., Richer, M. G., \& López, J. A. 2006b, A\&A, 455, 549

Rozas, M., Sabalisck, N., Beckman, J. E., \& Knapen, J. H. 1998, A\&A, 338, 15

Sabalisck, N., Tenorio-Tagle, G., Castañeda, H., \& Muñoz-Tuñon, C. 1995, ApJ, 444,200

Smith, M., \& Weedman, D. 1970, AJ, 161, 33

Steffen, W., \& López, J. A. 2006, Rev. Mex. Astron. Astrofis., 42, 99

Stone, J. M., \& Norman, M. L. 1992, ApJS, 80, 753

Tenorio-Tagle, G. 1979, A\&A, 71, 59

Tenorio-Tagle, G., Muñoz-Tunõn, C., \& Cid-Fernandes, R. 1996, ApJ, 456, 264

Terlevich, R., \& Melnick, J. 1981, MNRAS, 195, 839

Weaver, R., McCray, R., \& Castor, J. 1977, ApJ, 218, 377

Yang, H., Chu, Y.-H., Skillman, E. D., \& Terlevich, R. 1996, AJ, 112, 146 